\title{
The Three C's of Cethrene
}

\author{
Michal Juríček*
}

\begin{abstract}
Molecules that contain one or more unpaired electrons delocalized within a $\pi$-conjugated backbone are promising candidates for applications in spin electronics or simply 'spintronics'. Our group develops functional organic materials based on $\pi$-conjugated hydrocarbon molecules, where the electrons are unpaired either in the ground state or in the excited state that is low in energy and can be populated thermally. We aim to learn how to introduce and control a multitude of properties, namely, optical, chiroptical, magnetic, and conductive, in a bulk material made of these molecules, by manipulating spin interactions between the unpaired electrons. The first model system that was developed in our group is a hydrocarbon named cethrene, which has a diradicaloid singlet ground state and a low-lying triplet excited state. In this article, the structural parameters and their impact on the properties and reactivity of cethrene are discussed within the realm of the three C's that symbolize cethrene's C-shape, chirality, and chameleonic reactivity.
\end{abstract}

Keywords: Cethrene $\cdot$ Diradicaloid character $\cdot$ Helical chirality $\cdot$ Magnetic properties $\cdot$ Pericyclic ring-closure

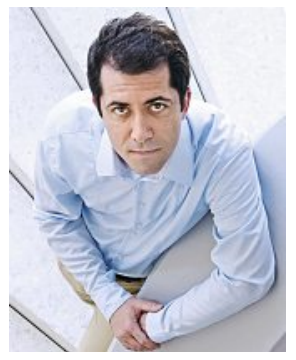

Michal Juríček was born and raised in Bojnice, Slovakia. He received his Master's degree in Organic Chemistry from the Comenius University in Bratislava in 2005. There under the supervision of Martin Putala, he studied how to conserve the configuration of binaphthyls during cross-coupling reactions. He then moved to The Netherlands to pursue his $\mathrm{PhD}$ at the Radboud University Nijmegen mentored by Alan E. Rowan. His doctoral work revolved around the chemistry of triazoles, which he employed in the design and synthesis of functional materials. In 2011, he joined the group of J. Fraser Stoddart at Northwestern University in Evanston,

${ }^{\star}$ Correspondence: Prof. M. Juríček E-mail: michal.juricek@chem.uzh.ch Department of Chemistry

University of Zurich

Winterthurerstrasse 190, CH-8057 Zurich
United States, as a postdoctoral scholar supported by Dutch fellowship Rubicon. He was synthesizing extended tetracationic cyclophanes to sequester hydrocarbon pollutants, mimic enzyme catalysis, and make molecular switches. In 2013, he returned to Europe and started his independent research career in Switzerland: first at the University of Basel as an Ambizione fellow of the Swiss National Science Foundation (SNSF) hosted by Marcel Mayor and, as of April 2017, as an Assistant Professor at the University of Zurich, where his research is supported by the European Research Council and SNSF. His group designs, synthesizes, and investigates functional organic molecules with delocalized spin densities.

\section{Introduction}

Organic molecules that contain one or more unpaired electrons and have a nonzero spin can serve as building blocks for the design of non-metal-based magnetic materials. ${ }^{[1]}$ In addition to cases where unpaired electrons in a molecule reside each on one atom only, molecules where unpaired electrons occupy multiple positions simultaneously are possible. ${ }^{[2]}$ Such molecules can be achieved by integrating unpaired electrons into a $\pi$-conjugated system, where all feasible resonance forms account for the distribution, or delocalization, of these electrons and thereby the spin. In spin-delocalized molecules, the spins of unpaired electrons "communicate' via the $\pi$-conjugated backbone and structural design allows not only to control the spin interactions between unpaired electrons, ${ }^{[3]}$ but also to generate different spin states and to control the energy gap between these states. ${ }^{[4]}$ Another feature of spin-delocalized systems is their ability to form $n$-electron multicenter bonds, or socalled pancake bonds, ${ }^{[5]}$ which are formed not between one but multiple pairs of atoms of the face-to-face oriented spin-delocalized systems. This bonding interaction is an attractive force that acts against electron repulsion between the $\pi$-systems ${ }^{[5 a]}$ and decreases the distance ${ }^{[6]}$ between the facially oriented units when compared to a regular $\pi-\pi$ stacking distance of $\sim 3.4$ A. A bulk material assembled from molecules with delocalized spin densities via pancake-bond formation can display a range of properties, including magnetism and conductivity, ${ }^{[7]}$ as well as bistability in response to external stimuli that allows to switch between two states, in which these properties differ substantially, for example, one state being IR transparent, paramagnetic, and insulating and the other one being IR absorbing, diamagnetic, and conducting. ${ }^{[7 \mathrm{c}]}$

The simplest case of a spin-delocalized hydrocarbon system is an allyl radical (Fig. 1, left panel, black), in which one unpaired electron is delocalized between two positions ( 1 and 3 ), the terminal carbon atoms. Attachment of an additional $\mathrm{sp}^{2}$-carbon atom gives either trimethylenemethane (TMM; red) or 1,3-butadiene (BD; blue), both $\mathrm{C}_{4} \mathrm{H}_{6}$ isomers, depending on whether this carbon atom is attached at the 2- (TMM) or 1-position (BD) of the allyl radical. ${ }^{[8]}$ The mode of connection is a game changer: while TMM has a non-Kekulé diradical structure containing two unpaired electrons and one double bond, BD has a Kekulé structure with two conjugated double bonds and no unpaired electrons. This simple exercise nicely illustrates the effect that the 'topology' of an $\mathrm{sp}^{2}$-carbon-atom backbone has on the electronic structure, which can be open-shell 
(TMM) or closed-shell (BD). Combining two TMM, two BD, or one TMM and one BD units together such that a six-membered ring with two methylene substituents is formed (Fig. 1, middle panel) leads to a series of para- (pQDM), meta- (mQDM), and ortho- (oQDM) quinodimethanes (Fig. 1 , right panel), also known as xylylenes. In this case, the combination of two units of the same kind gives Kekule quinoidal structures (either pQDM or oQDM), while only the combination of one TMM and one BD unit gives a non-Kekulé diradical structure (mQDM) with one Clar's sextet.

The allyl radical, TMM, BD, pQDM, $\mathrm{mQDM}$, and oQDM belong to the category of alternant hydrocarbons. ${ }^{[3,8,9]} \mathrm{A}$ $\pi$-conjugated hydrocarbon is alternant when stars (asterisks) can be placed on alternate $\mathrm{sp}^{2}$-carbon atoms with no stars being adjacent. When there is an unequal number of starred $\left(n_{\mathrm{s}}\right)$ and unstarred $\left(n_{\mathrm{u}}\right)$ atoms, the stars are placed such that $n_{\mathrm{s}}>$ $n_{\mathrm{u}}$. Alternant hydrocarbons are classified as even- and odd-alternant, where in the former case $n_{\mathrm{s}}=n_{\mathrm{u}}$ (BD, pQDM, and oQDM) and in the latter case $n_{\mathrm{s}}>n_{\mathrm{u}}$ (allyl radical, TMM, and mQDM). Even-alternant BD, pQDM, and oQDM have equal numbers of fully occupied bonding and empty antibonding orbitals, symmetrically distributed, and no non-bonding orbitals. As a result, each molecule can be represented by at least one Kekulé resonance structure in which all electrons are paired in form of conjugated double bonds. Even-alternant hydrocarbons can, however, also posses non-Kekulé diradical structures such as, for example, square cyclobutadiene $\left(n_{\mathrm{s}}=\right.$ $\left.n_{\mathrm{u}}=2\right)$ or tetramethyleneethane $\left(n_{\mathrm{s}}=n_{\mathrm{u}}=\right.$ 3). ${ }^{[9 b]}$ Odd-alternant hydrocarbons, such as allyl radical, TMM, and $\mathrm{mQDM}$, have also equal numbers of fully occupied bonding and empty antibonding orbitals, but also a set of non-bonding orbitals, the number of which equals to $n_{\mathrm{s}}-n_{\mathrm{u}}$. Each non-bonding orbital is occupied by one unpaired electron and the number $n_{\mathrm{s}}-n_{\mathrm{u}}$ is thus also equal to the number of unpaired electrons. In the case of these molecules, Kekulé resonance structures with all electrons paired cannot be drawn and only non-Kekulé resonance structures are possible (the positions of stars denote the positions where unpaired electrons can reside). In addition, the 'star' rule can be used to predict the ground state of a $\pi$-conjugated hydrocarbon. ${ }^{[9 a]}$ When $n>n_{\mathrm{u}}$, the ground state is of highest possible multiplicity (e.g., TMM has a triplet diradical ground state ${ }^{[10]}$ ) on account of the non-disjoint character of the non-bonding orbitals that have atoms in common. ${ }^{[9 b]}$ When $n_{\mathrm{s}}=n_{\mathrm{u}}$, the ground state is typically singlet (BD, pQDM, oQDM), even for

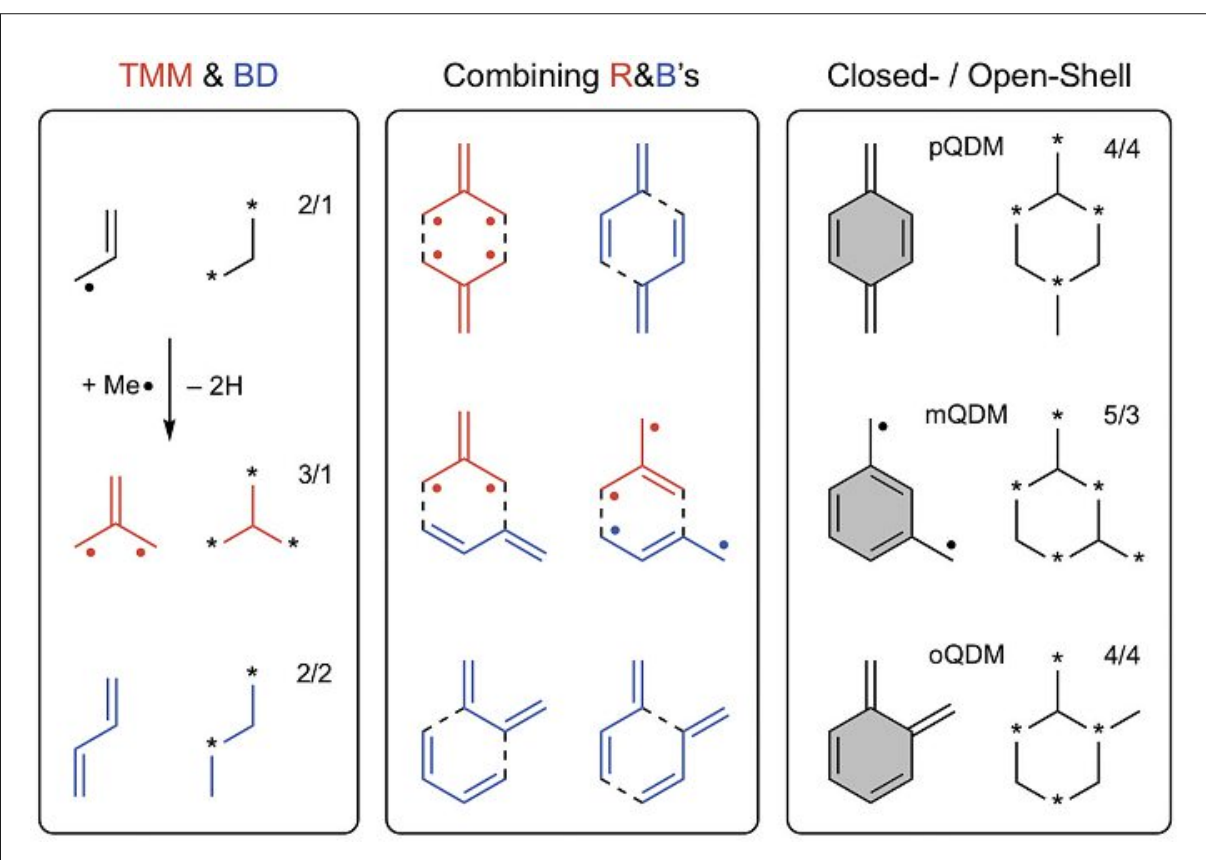

Fig. 1. (Left panel) The formation of trimethylenemethane (TMM; red) and 1,3-butadiene (BD; blue) from allyl radical (black) by introduction of one additional $\mathrm{sp}^{2}$-carbon atom at the 2- (TMM) or 1-position (BD). (Middle panel) Possible ways to combine two TMM, two BD, or one TMM and one BD units to form (right panel) para- (pQDM), meta- (mQDM), and ortho- (oQDM) quinodimethanes. BD, pQDM, and oQDM have Kekule structures with all $\pi$-electrons paired. TMM and mQDM have non-Kekulé structures with two unpaired $\pi$-electrons. All molecules are alternant hydrocarbons, that is, stars (asterisks) can be placed on alternating carbon atoms with no two stars adjacent. The difference between the number of starred $\left(n_{\mathrm{s}}\right.$; first number) and unstarred $\left(n_{\mathrm{u}}\right.$; second number) carbon atoms equals to the number of unpaired electrons. If $n_{\mathrm{s}}=n_{\mathrm{u}}$, the system is even-alternant. If $n_{\mathrm{s}} \neq n_{\mathrm{u}}$, the system is odd-alternant and then the atoms are starred such that $n_{\mathrm{s}}>n_{\mathrm{u}}$; this way, the stars denote all the possible positions of the unpaired electron(s).

diradical systems such as square cyclobutadiene. ${ }^{[11]}$ Here, the non-bonding orbitals are disjoint, that is, they do not have atoms in common, and to a first approximation the lowest singlet and triplet states have the same energy. Because of dynamic spin polarization that selectively stabilizes nonuniform distribution of electron spin in the non-bonding orbitals, however, diradical molecules with $n=n$ typically have a singlet ground state. ${ }^{\mathrm{s}}{ }^{11 \mathrm{a}]}$

The BD and TMM units can be used to construct even larger systems. Fig. 2 shows three out of five possible isomers ${ }^{[4]}$ with a molecular formula $\mathrm{C}_{28} \mathrm{H}_{16}$ composed of seven fused six-membered rings. In the case of [7]zethrene and cethrene, seven BD units can be used to assemble the $\pi$-conjugated core of both compounds, while in the case of [7]uthrene, one BD unit needs to be replaced by a TMM unit. As a result, all electrons are paired in [7]zethrene and cethrene to give a system with 14 conjugated double bonds. In [7]uthrene, two electrons remain unpaired and in resonance with 13 conjugated double bonds. Also in this case, the star rule can be applied, leading to $n_{\mathrm{s}}=n_{\mathrm{u}}=14$ for [7]zethrene and cethrene and $n_{\mathrm{s}}=15$ and $n_{\mathrm{u}}=13$ for [7]uthrene. The outcome is a Kekulé system with a singlet ground state in the former cases and a non-Kekulé diradical system with a triplet ground state in the latter case. Out of five possible isomers, only [7]zethrene and [7] uthrene were known ${ }^{[3,12]}$ prior to 2016, when we reported ${ }^{[13]}$ the synthesis and properties of the first derivative of cethrene. In this account, the structure-property relationship in cethrene is discussed within three sections where the letter ' $C$ ' symbolizes cethrene's C-shape, chirality, and chameleonic reactivity.

\section{'C' for C-Shape}

The names of the homologs of the isomeric series $\mathrm{C}_{28} \mathrm{H}_{16}$ are derived $[3,13,14]$ from their molecular shape (Fig. 3): the Z-shape of [7]zethrene (dibenzo[de,op]pentacene), the U-shape of [7]uthrene (dibenzo[de,jk] pentacene), and the $\mathrm{C}$-shape of cethrene (dibenzo[ $p q, u v]$ pentaphene). The number in the square brackets denotes the number of fused six-membered rings. In cethrene, this number is omitted as cethrene is the first homolog of the cethrene series featuring an $[n]$ helicene subunit (in the case of parent cethrene, $n=5$ ). The first homologs of the zethrene and uthrene series featuring an $[n]$ acene subunit are zethrene and uthrene $(n=4$; total number of rings $=6$ ), respectively. In this respect, [6]cethrene would have an identical structure as [6]uthrene, therefore, [7]cethrene is the parent molecule. These isomers can also be obtained by fusing two phenalenyl units 


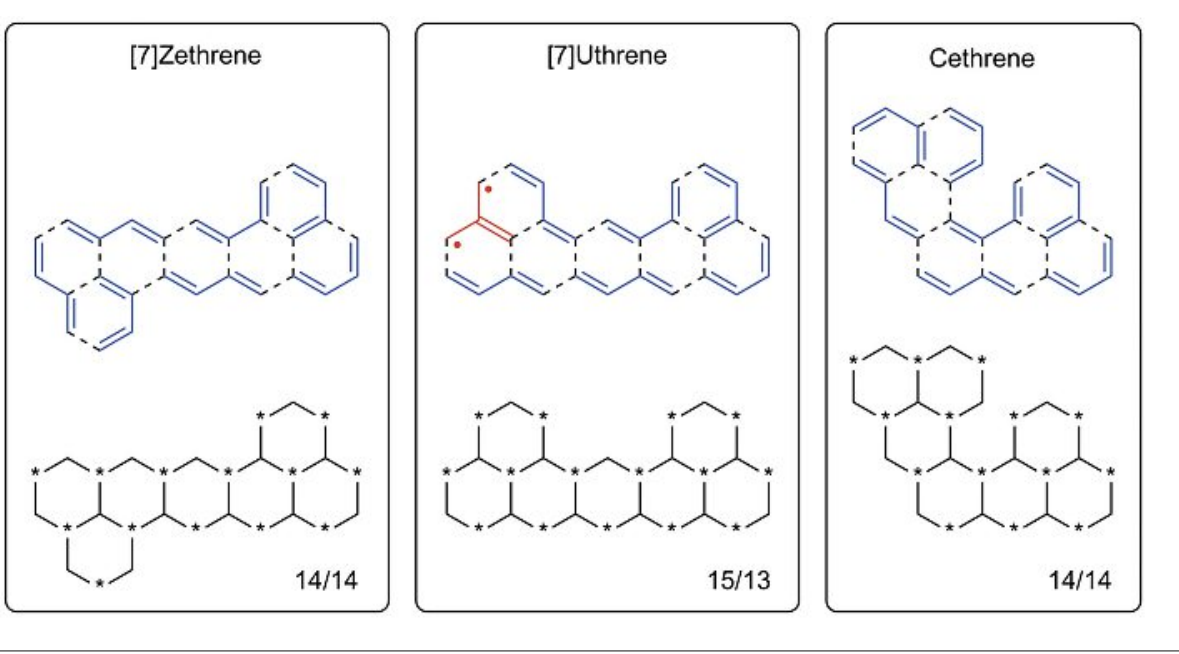

Fig. 2. (Top) Assembling the structure of [7]zethrene (left panel) and cethrene (right panel) from seven BD units (blue) and the structure of [7] uthrene (middle panel) from six BD (blue) and one TMM (red) units. (Bottom) For [7]zethrene and cethrene, $n_{\mathrm{s}}-n_{\mathrm{u}}=14-14=0$, which leads to even-alternant Kekulé structures with all $\pi$-electrons paired. For [7] uthrene, $n_{\mathrm{s}}-n_{\mathrm{u}}=15-13=2$, which leads to an odd-alternant non-Kekulé structure with two unpaired $\pi$-electrons. See caption to Fig. 1 for the definition of $n_{\mathrm{s}}$ and $n_{\mathrm{u}}$.

to a central benzene ring. Depending on the fusion mode, either a Kekulé (para-l para-fusion in [7]zethrene and para-/ ortho-fusion in cethrene) or a non-Kekulé (meta-/meta-fusion in [7]uthrene) system is obtained (Fig. 3, top panel). The Kekulé systems possess a quinoidal structure featuring a pQDM subunit (in blue) and two Clar's sextets (gray-filled rings). The nonKekulé system has a diradical structure featuring a mQDM subunit (in red) and three Clar's sextets (gray- and red-filled rings). A diradical resonance structure can also be drawn for the Kekulé systems. In such a case, an additional Clar's sextet (red-filled ring) is obtained.

Kekulé systems such as [7]zethrene and cethrene, where the quinoidal pQDM subunit disrupts the aromaticity of three out of seven rings and the diradical resonance structure displays an additional Clar's sextet, typically have a small energy gap (i) between the highest occupied (HOMO) and the lowest unoccupied (LUMO) molecular orbitals and (ii) between the singlet ground state and the first excited triplet state (Fig. 4). ${ }^{[4]}$ On account of the small HOMO-LUMO gap, these molecules typically absorb light $\left(\mathrm{S}_{0}-\mathrm{S}_{1}\right.$ transition) in the visible region $\left(\lambda_{\max }([7]\right.$ zethrene $)=$ $586 \mathrm{~nm},{ }^{[3]} \lambda_{\text {max }}$ (cethrene) $\left.=665 \mathrm{~nm}^{[13 \mathrm{~b}]}\right)$. For a comparison, coronone that is composed of seven fused rings and does not have a quinoidal structure displays a much larger HOMO-LUMO gap and absorbs light mostly in the UV region. As a result of the small singlet-triplet (ST) gap, the triplet excited state can sometimes be populated thermally, which gives rise to magnetic properties of these Kekulé systems. Depending on the magnitude of the
ST gap, thermal equilibrium leads to the population of the triplet state according to the Boltzmann distribution, and an electron paramagnetic resonance (EPR) spectrum of the species in the triplet excited state can be recorded. ${ }^{[2 b, 4]}$ Considering the sensitivity of the EPR instrument and a concentration of the sample (typically in the range of $10^{-4}$ to $10^{-5} \mathrm{M}$ ), an ST gap of roughly less than $8 \mathrm{kcal} \mathrm{mol}^{-1}$ is needed. If an EPR signal is observed, variable-temperature EPR measurements can be performed (the intensity of the signal decreases with decreasing temperature) and the data can be fitted by using the Bleaney-Bowers equation ${ }^{[15]}$ to obtain an experimental value of the ST gap. Another consequence of the low ST gap is that broadening of the signals in the ${ }^{1} \mathrm{H}$ NMR spectrum can be observed. This broadening is also temperature-dependent and increases with increasing temperature, that is, increasing population of the triplet state. ${ }^{\text {[7b, 12a] }}$

The very small HOMO-LUMO gap in quinoidal Kekulé systems needs to be considered for a correct description of their ground states (blue square in Fig. 4; $a=\mathrm{HOMO}, b=\mathrm{LUMO}$ ), which requires an admixture of a doubly excited configuration $\left(a^{0} b^{2}\right)$ into a ground configuration $\left(a^{2} b^{0}\right)$ or, loosely speaking, part of the electrons from the HOMO are placed into the LUMO to minimize electron repulsion. $[2 \mathrm{~b}, \mathrm{~d}, 16]$ Because the bond order is decreased and both the HOMO and the LUMO are occupied by fewer than two electrons each, the singlet ground state can be described as 'diradicaloid' . ${ }^{[16 b]}$ It is important to note that the term diradicaloid does not infer the presence of unpaired electrons, as can be easily mistaken, but the fact that the fron-
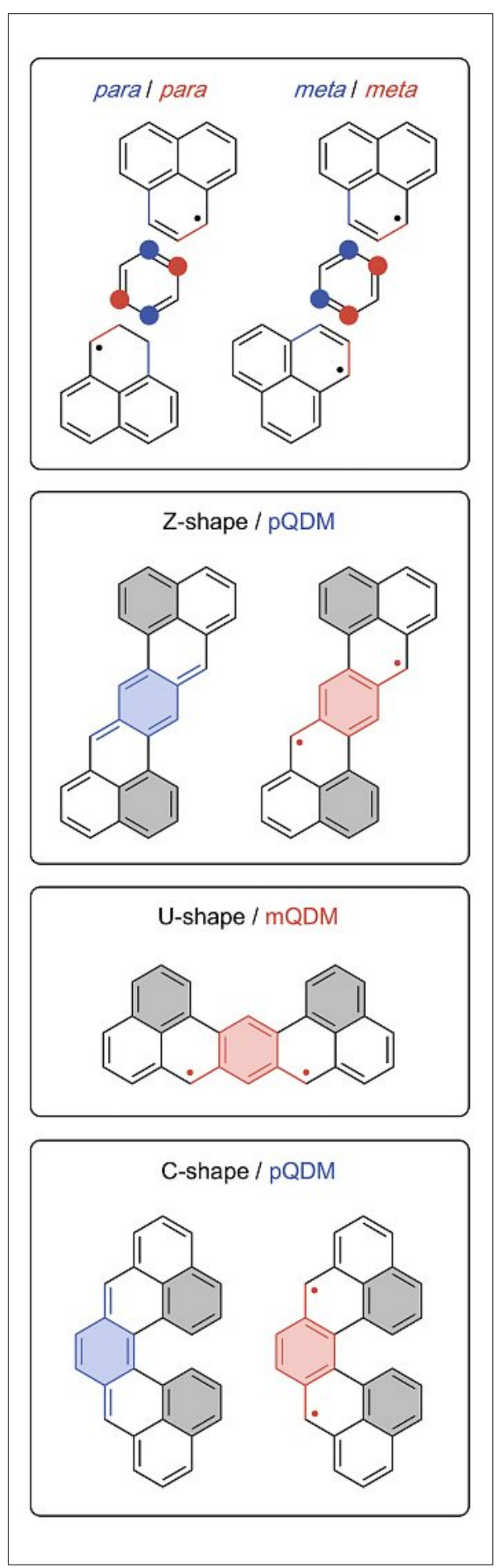

Fig. 3. (Top panel) Two examples of how two phenalenyl units (three rings) can be fused to a central benzene ring. In the case of Z-shaped [7]zethrene (second from top panel) and C-shaped cethrene (bottom panel), the quinoidal resonance structures shown on the left (pQDM subunit is highlighted in blue) display two Clar's sextets (gray-filled rings), while the diradical resonance structures (pQDM subunit is highlighted in red) shown on the right display one additional Clar's sextet (red-filled ring). Molecules with these structural features typically display small HOMO-LUMO and singlet-triplet (ST) energy gaps (see Fig. 4). In the case of U-shaped [7]uthrene (second from bottom panel; mQDM subunit is highlighted in red), only diradical resonance structures with a maximum number of Clar's sextets equal to three are possible. 
Fig. 4. Quinoidal molecules pQDM, oQDM, [7]zethrene, and cethrene display small HOMO-LUMO energy gaps ( $a=\mathrm{HOMO}, b=$ LUMO). The correct description of their ground states (blue square) therefore requires an admixture of a doubly excited configuration $\left(a^{0} b^{2}\right)$ into a ground configuration $\left(a^{2} b^{0}\right)$ or, loosely speaking, part of the electrons from the $\mathrm{HOMO}$ are placed into the LUMO to minimize electron repulsion. Because the bond order is decreased and both the HOMO and the LUMO are occupied by less than two electrons each, the singlet ground state can be described as diradicaloid. A common feature of diradicaloid compounds is that the triplet excited state $\left(a^{1} b^{1}\right.$; red square) is relatively low in energy, which can make it thermally accessible. The thermal equilibrium between the diradicaloid singlet ground state (blue) and the triplet excited state (red) is shown for pQDM.

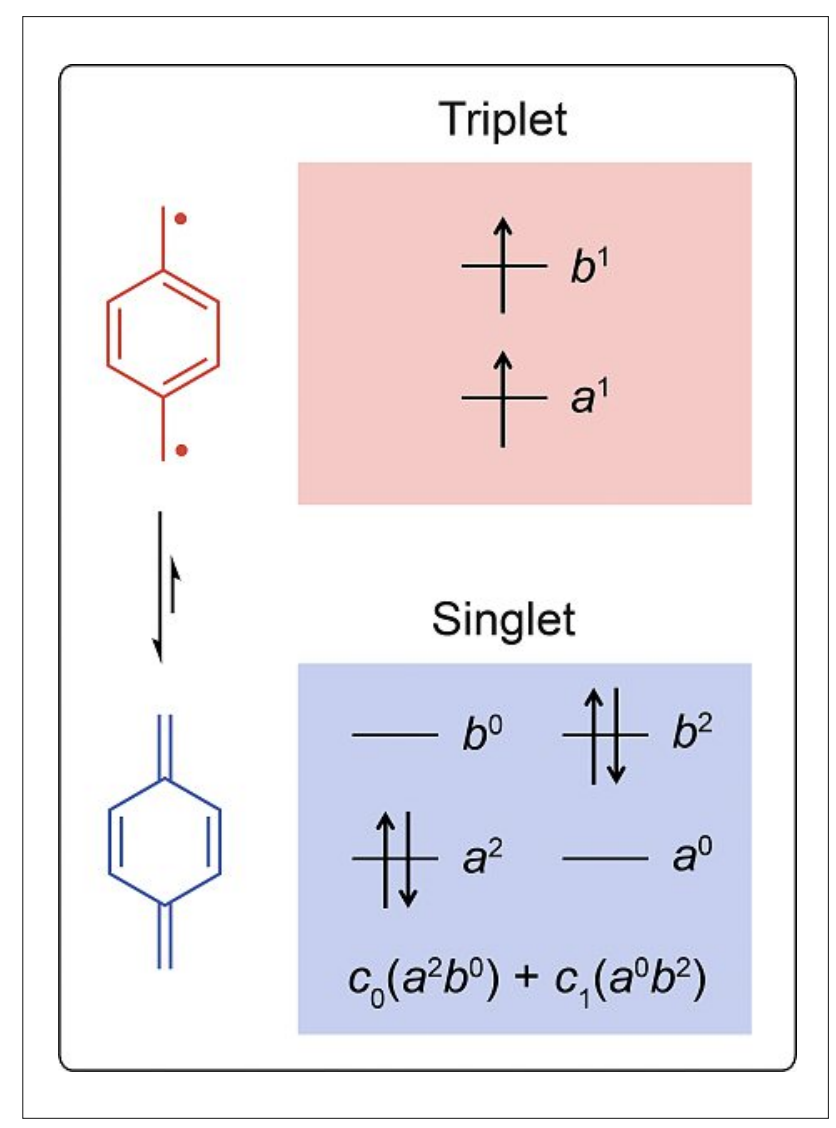

tier molecular orbitals are not fully occupied although the electrons are paired. The diradicaloid character of molecules represents a challenge for computational methods. ${ }^{[2 \mathrm{~b}]}$ For example, DFT is a singlereference method and the ground state of a diradicaloid molecule can be either optimized by using a spin-restricted wavefunction (electrons are only in the HOMO) or a spin-unrestricted broken-symmetry wavefunction. Because none of these approaches is ideal, it is important to compare the results of the two with experiment. Multi-reference methods such as CASPT2 are better suited, however, they are significantly more time-consuming, in particular for systems as large as [7]zethrene and cethrene. These methods can also provide information on the occupancy of the HOMO and the LUMO. In addition, the partial occupancy of the frontier molecular orbitals endows diradicaloid molecules with the ability to form pancake bonds. For example, Kubo et al. demonstrated[7b] that a diradicaloid system composed of two phenalenyl units forms in the solid state an infinite stack, where each phenanenyl subunit forms a pancake bond with a phenalenyl subunit of the neighboring molecule. In this unique pancake-bonded system, the unpaired electrons of the phenalenyl subunits interact intramolecularly through the backbone of the molecule and, at the same time, intermolecularly through space between the molecules. As a result, the bulk material displayed lower HOMO-LUMO and ST gaps, when compared to those of single diradicaloid monomer.

\section{'C' for Chirality}

Our interest in synthesizing and studying cethrene was inspired by the work of Kubo et al., who demonstrated[7b] that intermolecular through-space interactions can affect the spin coupling between unpaired electrons. In contrast to planar [7]zethrene and [7]uthrene, which possess a pentacene subunit (Fig. 5, top panel, redfilled rings), the structure of cethrene features a [5]helicene subunit (Fig. 5, bottom panel, blue-filled rings). Because of steric interactions between the hydrogen atoms in the fjord region, cethrene is not planar. Instead, it adopts a helically twisted geometry with a $C_{2}$ symmetry and displays axial chirality. ${ }^{[17]}$ In this system, the electrons can 'communicate' simultaneously via the backbone and through space within a molecule. Thus, we were intrigued to find out how the through-space interactions would affect the HOMO-LUMO and ST gaps of cethrene when compared to the planar isomer [7]zethrene, which also has a singlet diradicaloid ground state and a low-lying triplet excited state. On account of the helical geometry, cethrene exists in the form of two enantiomers. To demonstrate that cethrene is chiral, we successfully separated the enantiomers of its dihydro-precursor, which displayed mirror-image Cotton ef- fects in the CD spectra. Kinetic measurements were performed to determine the energy barrier of enantiomerization at 298 $\mathrm{K}\left(\sim 25 \mathrm{kcal} \mathrm{mol}^{-1}\right)$, which matched that of parent [5] helicene $\left(\sim 24 \mathrm{kcal} \mathrm{mol}^{-1}\right)$. $^{[13 \mathrm{~b}]}$ Note: in the original work, a diphenyl derivative of cethrene was studied.

Fig. 5 shows the HOMO and the LUMO of [7]zethrene and cethrene obtained from the Hückel molecular orbital (HMO) analysis. In both cases, the shape of the HOMO nicely reflects the pQDM subunit, which indicates that both diradicaloid molecules are best represented by the quinoidal resonance structures (Fig. 3 , left). These are in good agreement with both the bond lengths and NICS profiles that were obtained from DFT. ${ }^{[13 b]}$ The diradical resonance structures (Fig. 3, right) best represent the excited state of these molecules. In contrast to planar [7]zethrene, however, an antibonding ( $a b)$ and a bonding (b) interaction arise in cethrene within the HOMO and the LUMO, respectively, because of the helical twist (Fig. 5, bottom panel). The antibonding interaction increases the energy of the HOMO and the bonding interaction decreases the energy of the LUMO. As a result, cethrene displays a smaller HOMO-LUMO gap, when compared to [7]zethrene. This feature can be observed experimentally by comparison of the absorption spectra of both compounds, where absorption maximum of cethrene $\left(665 \mathrm{~nm}^{[13 \mathrm{~b}]}\right)$ is shifted significantly to the red when compared to that $\left(586 \mathrm{~nm}^{[3]}\right)$ of [7]zethrene.

The through-space orbital interactions also affect the ST gap, and while [7]zethrene is EPR silent, ${ }^{[12 a]}$ a signal of the thermally populated triplet state was observed[13b] in the EPR spectrum of cethrene at room temperature. Variabletemperature experiments described above allowed determination of the ST gap ( 5.6 $\mathrm{kcal} \mathrm{mol}^{-1}$ ), which is in an excellent agreement with DFT ( $\left.5.9 \mathrm{kcal} \mathrm{mol}^{-1}\right)$. Compared to [7]zethrene (ST gap of $8.9 \mathrm{kcal} \mathrm{mol}^{-1}$ by $\left.\mathrm{DFT}^{[13 \mathrm{~b}]}\right)$, the ST gap of cethrene is markedly lower. The decrease of the ST gap can be attributed to the through-space orbital interactions in cethrene and rationalized as follows: in the singlet ground state, the electrons occupy mainly the HOMO (occupation numbers obtained by CASSCF $(12,12)$ are 1.85 for HOMO and 0.15 for LUMO), while in the triplet state, the HOMO and the LUMO are occupied by one electron each. The singlet ground state therefore suffers more from the antibonding interaction in the HOMO than the triplet state and its energy is increased relative to the triplet state. Consequently, the ST gap is decreased. This rational was further supported by computing ${ }^{[13 b]}$ the ST gap in a hypothetical planar cethrene $(8.0$ kcal $\mathrm{mol}^{-1}$ by DFT), which is similar to 

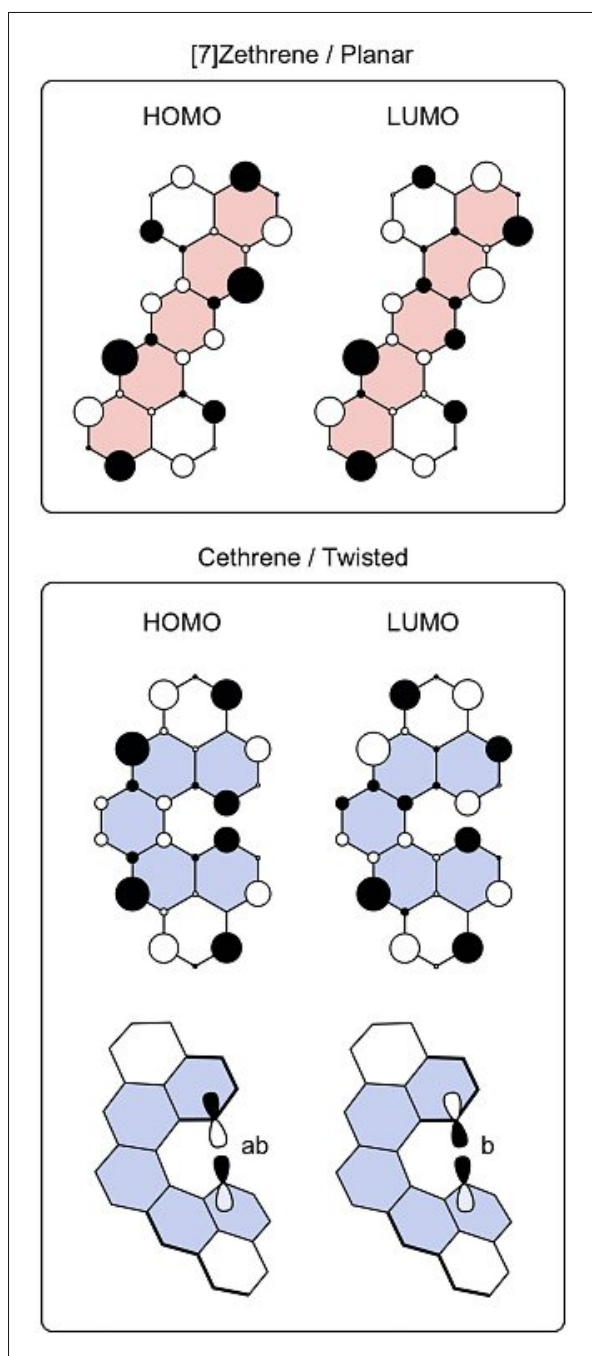

Fig. 5. The HOMO and the LUMO of planar [7]zethrene (top panel; pentacene subunit is highlighted in red) and helically twisted cethrene (bottom panel; [5]helicene subunit is highlighted in blue) obtained from Hückel molecular orbital (HMO) analysis. In cethrene, an antibonding (ab) and a bonding (b) intramolecular through-space interactions arise within the HOMO and the LUMO, respectively, on account of the helical twist. Because in the singlet ground state of diradicaloids the occupancy of the HOMO is larger than that of the LUMO $\left(c^{0}>c^{1}\right)$ and in the triplet excited state both orbitals are equally occupied by one electron each, in cethrene the singlet state suffers more from the antibonding interaction in the HOMO than the triplet state. As a result, the singlet state is destabilized relative to the triplet state and the singlet-triplet (ST) energy gap of cethrene $\left(\sim 5.6 \mathrm{kcal} \mathrm{mol}^{-1}\right)$ is decreased when compared to that $\left(8.9 \mathrm{kcal} \mathrm{mol}^{-1}\right)$ of planar [7]zethrene.

that of planar [7]zethrene, where throughspace orbital interactions are not possible. The antibonding and bonding orbital interactions also become obvious when one looks at the distance between the carbon atoms in the fjord region of cethrene (geometries optimized by DFT), which is larger in the singlet ground state $(3.03 \AA)$ than in the triplet excited state $(2.93 \AA)$.
These findings illustrate that introduction of intramolecular through-space orbital interactions is a useful tool to modulate the HOMO-LUMO and ST gaps in diradicaloid systems. In helical systems such as cethrene, fine-tuning of these gaps can be further achieved by controlling the fjord carbon-carbon distance through steric effects, for example, by installment of suitable substituents at these positions.

\section{'C' for Chameleon}

During our investigations of the properties of a diphenyl derivative of cethrene, we noticed that this compound is not stable and that it undergoes a transformation to a planar hydrocarbon within just a few hours at room temperature. ${ }^{[13 b]}$ We hypothesized that the first step of this transformation is an electrocyclic ring-closure leading to a closed-form of cethrene (Scheme 1, bottom panel, upper scheme, $\mathrm{R}=\mathrm{Ph}$ ), an intermediate that is subsequently oxidized by traces of oxygen or an excess of oxidant $p$-chloranil to give the final flat product. Because of steric constrains imposed by the helical geometry of cethrene, the conrotatory ring-closure is favored over a disrotatory process. According to the Woodward-Hoffmann rules, ${ }^{[18]}$ however, the thermal conrotatory process should be symmetry-forbidden on account of the antibonding interaction in the HOMO (see Fig. 5), which is an analogous case to the electrocyclic ring-closure of 1,6-dimethyl1,3,5-hexatriene (Scheme 1, top panel). This mismatch caught our interest and we investigated this process in more detail. [19]

Kinetic studies were performed ${ }^{[19]}$ in order to determine the activation barrier of the thermal process, which was found to be $\sim 14 \mathrm{kcal} \mathrm{mol}^{-1}$. This value is similar to that $\left(\sim 16 \mathrm{kcal} \mathrm{mol}^{-1}\right)$ obtained ${ }^{[20 \mathrm{a}]}$ by Kubo et al. for an analogous biphenalenylidene

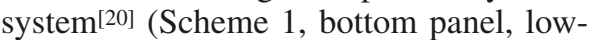
er scheme). Both activation barriers are surprisingly low, considering the forbidden nature of the processes. What is even more surprising is that these barriers are lower than barriers of many electroyclic processes that are thermally allowed. The reason for the ease of the thermal conrotatory process is the diradicaloid character of cethrene. Since both the HOMO and the LUMO are partially occupied in the ground state, the activation barrier is decreased and the electrocyclic ring-closure of cethrene is 'allowed' both thermally and photochemically in a conrotatory mode. This chameleonic character is different from other pericyclic reactions that proceed either thermally or photochemically in a given rotatory mode and places cethrene among a rare type of molecules, such as biphenalenylidene ${ }^{[20]}$ and the first example of this kind, pleiadene, reported by Michl et al. [21] Our calculations showed[19] that the thermally allowed disrotatory ring-closure of cethrene is thermoneutral or slightly endothermic and its activation barrier is higher by $\sim 4 \mathrm{kcal} \mathrm{mol}^{-1}$ than that of the exothermic $C_{2}$-symmetric conrotatory process $\left(\sim 23 \mathrm{kcal} \mathrm{mol}^{-1}\right)$. A variety of DFT and wavefunction-based methods gave comparable results, however, all methods failed to match the experimental value $\left(\sim 14 \mathrm{kcal} \mathrm{mol}^{-1}\right)$. Diradicaloid systems such as cethrene, biphenalenylidene, and pleiadene therefore represent a test for theoretical methods to find the most favorable reaction pathways.

\section{'C'oncluding Remarks}

The conrotatory electrocyclic ring-closure of cethrene was shown ${ }^{[19]}$ to proceed also photochemically upon irradiation at $660 \mathrm{~nm}$ (Scheme 1, bottom panel, upper scheme, $\mathrm{R}=\mathrm{Ph}$ ), in accord with the orbital-symmetry considerations, ${ }^{[18]}$ where the symmetry of the LUMO governs the photochemical process. Interestingly, the photochemical ring-closure of analogous biphenalenylidene did not proceed upon irradiation at $700 \mathrm{~nm}$ in the solvent matrix. This process could, however, be reversed upon irradiation at $365 \mathrm{~nm}$ as shown[20a] by Kubo et al. (Scheme 1, bottom panel, lower scheme). These and our results indicate that this type of helical diradicaloid molecules could serve as switches operated solely by light, where the open and the closed form differ substiantially in their optical (HOMO-LUMO gap), magnetic (ST gap), and chiroptical (degree of twist) properties. These molecules therefore hold potential as molecular building blocks of non-metallic materials, where bulk magnetic and conducting properties, archetypal for metals, can be turned on and off by light. Finally, the helical geometry of the molecular building blocks endows them with a potential to be employed as components of enantiomerically pure forms of these materials, which could serve as novel model systems for investigation of effects that arise from the interplay of magnetism and chirality.

\section{Acknowledgements}

I would like to thank all former and current group members who contributed immeasurably to the development of this research and continue to do so: Dr. Prince Ravat, Dr. Tomáš Šolomek, Peter Ribar, Rahel Hinkelmann, David Steinebrunner, Laurent Jucker, Tomáš Pastierik, Daniel Čavlović, Mohebodin Karbasiyoun, and Annika Bernhardt. I would also like to thank Prof. Dr. Marcel Mayor, who generously hosted and supported our group at the University of Basel during the Ambizione program (2013-2017). This project has re- 
Scheme 1. (Top panel) In accordance with the Woodward-Hoffmann rules, the electrocyclic ring-closure of 1,6-dimethyl-1,3,5-hexatriene proceeds photochemically in a conrotatory mode (red arrows; governed by the symmetry of the LUMO) to give the trans-product and thermally in a disrotatory mode (blue arrows; governed by the symmetry of the HOMO) to give the cis-product. (Bottom panel) The electrocyclic ringclosure of cethrene (up) has a chameleonic nature as it proceeds in a conrotatory mode both thermally and photochemically. The formally 'forbidden' thermal process is 'allowed' because the LUMO is partially occupied in the ground state on account of the diradicaloid character of cethrene. Analogous diradicaloid system biphenalenylidene (down) was also shown to undergo the thermal conrotatory electrocyclic ring-closure with an activation barrier $(\sim 16$

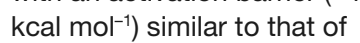
cethrene $\left(\sim 14 \mathrm{kcal} \mathrm{mol}^{-1}\right)$. Although the photochemical process is allowed also in this case, it did not proceed upon irradiation by light in the solvent matrix. The reversed electroyclic ring-opening process occurred upon irradiation.
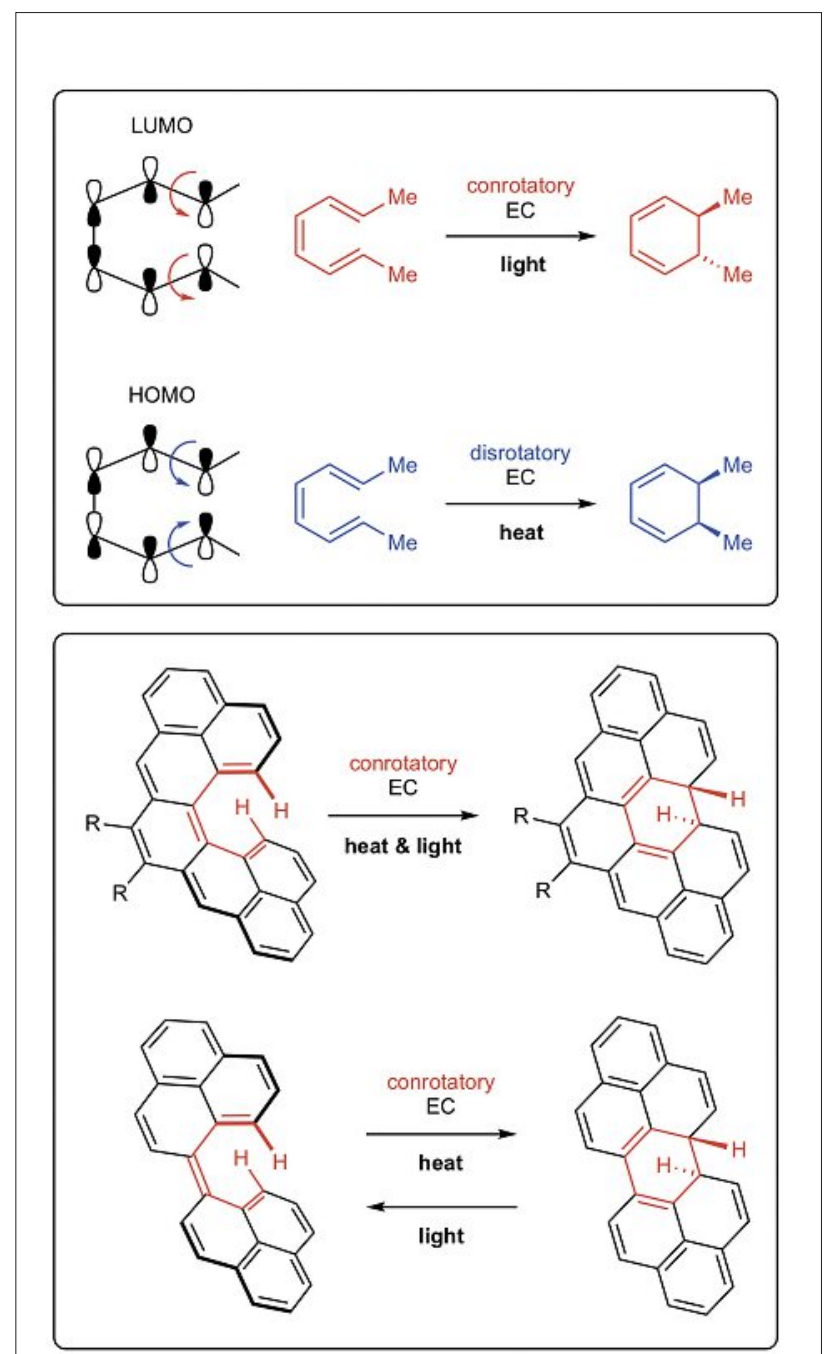

ceived funding from the European Research Council (ERC) under the European Union's Horizon 2020 research and innovation programme (Grant Agreement No. 716139) and from the Swiss National Science Foundation (SNSF, PP00P2_170534).

[1] a) 'Stable Radicals: Fundamentals and Applied Aspects of Odd-Electron Compounds', Ed.: R. G. Hicks, Wiley, Chichester, 2010; b) 'Carbon Based Magnetism', Eds.: T. Makarova, F. Palacio, Elsevier, Amsterdam, 2006.

[2] a) T. Kubo, Chem. Rec. 2015, 15, 218; b) M. Abe, Chem. Rev. 2013, 113, 7011; c) Y. Morita, S. Suzuki, K. Sato, T. Takui, Nat. Chem. 2011, 3, 197; d) L. Salem, C. Rowland, Angew. Chem. Int. Ed. 1972, 11, 92.
[3] E. Clar, I. A. Macpherson, Tetrahedron 1962 , $18,1411$.

[4] Z. Sun, Z. Zeng, J. Wu, Acc. Chem. Res. 2014, 47,2582

[5] a) Z.-H. Cui, H. Lischka, H. Z. Beneberu, M. Kertesz, J. Am. Chem. Soc. 2014, 136, 5539; b) Z. Mou, K. Uchida, T. Kubo, M. Kertesz, J. Am. Chem. Soc. 2014, 136, 18009; c) Z.-H. Cui, H. Lischka, H. Z. Beneberu, M. Kertesz, J. Am. Chem. Soc. 2014, 136, 12958; d) Y.-H. Tian, J. Huang, M. Kertesz, Phys. Chem. Chem. Phys. 2010, 12, 5084; e) J. S. Miller, J. J. Novoa, Acc. Chem. Res. 2007, 40, 189; f) S. Suzuki, Y. Morita, K. Fukui, K. Sato, D. Shiomi, T. Takui, K. Nakasuji, J. Am. Chem. Soc. 2006, 128, 2530; g) Y. Takano, T. Taniguchi, H. Isobe, T. Kubo, Y. Morita, K. Yamamoto, K. Nakasuji, T. Takui, K. Yamaguchi, J. Am. Chem. Soc. 2002, 124, 11122.

[6] K. Goto, T. Kubo, K. Yamamoto, K. Nakasuji, K. Sato, D. Shiomi, T. Takui, M. Kubota, T.
Kobayashi, K. Yakusi, J. Y. Ouyang, J. Am. Chem. Soc. 1999, 121, 1619.

[7] a) S. K. Pal, M. E. Itkis, F. S. Tham, R. W. Reed, R. T. Oakley, R. C. Haddon, Science 2005, 309, 281; b) T. Kubo, A. Shimizu, M. Sakamoto, M Uruichi, K. Yakushi, M. Nakano, D. Shiomi, K. Sato, T. Takui, Y. Morita, K. Nakasuji, Angew. Chem. Int. Ed. 2005, 44, 6564; c) M. E. Itkis, X. Chi, A. W. Cordes, R. C. Haddon, Science 2002, 296, 1443.

[8] H. C. Longuet-Higgins, J. Chem. Phys. 1950, $18,265$.

[9] a) W. T. Borden, J. Org. Chem. 2011, 76, 2943; b) W. T. Borden, E. R. Davidson, J. Am. Chem. Soc. 1977, 99, 4587; c) C. A. Coulson, G. S. Rushbrooke, Proc. Camb. Phil. Soc. 1940, 36 , 193.

[10] P. Dowd, Acc. Chem. Res. 1972, 5, 242

[11] a) W. T. Borden, J. Am. Chem. Soc. 1975, 97, 5968; b) G. Maier, Angew. Chem. Int. Ed. Engl. 1974, 13, 425

[12] a) Y. Li, W.-K. Heng, B. S. Lee, N. Aratani, J. L. Zafra, N. Bao, R. Lee, Y. M. Sung, Z. Sun, K.W. Huang, R. D. Webster, J. T. López Navarrete, D. Kim, A. Osuka, J. Casado, J. Ding, J. Wu, J. Am. Chem. Soc. 2012, 134, 14913; b) Y. Li, K. W. Huang, Z. Sun, R. D. Webster, Z. Zeng, W. Zeng, C. Chi, K. Furukawa, J. Wu, Chem. Sci. 2014, 5, 1908.

[13] a) P. Ravat, T. Šolomek, P. Ribar, M. Juríček, Synlett 2016, 27, 1613; b) P. Ravat, T. Šolomek, M. Rickhaus, D. Häussinger, M. Neuburger, M. Baumgarten, M. Juríček, Angew. Chem. Int. Ed. 2016, $55,1183$.

[14] M. Melle-Franco, Chem. Commun. 2015, 51, 5387.

[15] a) B. Bleaney, K. D. Bowers, Proc. R. Soc. London, Ser. A 1952, 214, 451; b) P. Ravat, M. Baumgarten, Phys. Chem. Chem. Phys. 2015, 17, 983 .

[16] a) J. Michl, V. Bonačić-Koutecký, Tetrahedron 1988, 44, 7559; b) J. Kolc, J. Michl, J. Am. Chem. Soc. 1973, 95, 7391; c) J. Michl, Mol. Photochem. 1972, 4, 257.

[17] M. Rickhaus, M. Mayor, M. Juríček, Chem. Soc. Rev. 2016, 45, 1542.

[18] a) R. B. Woodward, R. Hoffmann, 'The Conservation of Orbital Symmetry', Verlag Chemie, Weinheim, 1970; b) I. Fleming, 'Molecular Orbitals and Organic Chemical Reactions', Wiley, Chichester, 2010, pp. 253368 ; c) R. B. Woodward, R. Hoffmann, Angew. Chem. Int. Ed. 1969, 8, 781; d) R. B. Woodward, R. Hoffmann, J. Am. Chem. Soc. 1965, 87, 295.

[19] T. Šolomek, P. Ravat, Z. Mou, M. Kertesz, M. Juríček, J. Org. Chem. 2018, 83, 4769.

[20] a) K. Uchida, S. Ito, M. Nakano, M. Abe, T. Kubo, J. Am. Chem. Soc. 2016, 138, 2399; b) S. Pogodin, I. Agranat, J. Am. Chem. Soc. 2003, 125, 12829.

[21] a) R. P. Steiner, J. Michl, J. Am. Chem. Soc 1978, 100, 6413; b) J. Kolc, J. Michl, J. Am. Chem. Soc. 1970, 92, 4147. 